SPIN-06-37, ITP-UU-06-47

\title{
Problems and hopes in nonsymmetric gravity $\ddagger$
}

\author{
Tomas Janssen ${ }^{1}$ and Tomislav Prokopec ${ }^{2}$ \\ Institute for Theoretical Physics, University of Utrecht, Leuvenlaan 4, Postbus \\ 80.195, 3508 TD Utrecht, The Netherlands \\ E-mail: ${ }^{1}$ T. Janssen2@phys .uu .nl,${ }^{2}$ T.Prokopec@phys . uu .nl
}

\begin{abstract}
We consider the linearized nonsymmetric theory of gravitation (NGT) within the background of an expanding universe and near a Schwarzschild mass. We show that the theory always develops instabilities unless the linearized nonsymmetric lagrangian reduces to a particular simple form. This form contains a gauge invariant kinetic term, a mass term for the antisymmetric metric-field and a coupling with the Ricci curvature scalar. This form cannot be obtained within NGT. Based on the linearized lagrangian we know to be stable, we consider the generation and evolution of quantum fluctuations of the antisymmetric gravitational field ( $B$-field) from inflation up to the present day. We find that a $B$-field with a mass $m \propto 0.03\left(H_{I} / 10^{13} \mathrm{GeV}\right)^{4} \mathrm{eV}$ is an excellent dark matter candidate.

PACS numbers: 04.20.-q, 04.90.+e, 98.80.-k
\end{abstract}

Submitted to: J. Phys. A: Special Issue IRGAC 2006

$\ddagger$ As presented by the authors at the 2nd International Conference on Quantum Theories and Renormalization Group in Gravity and Cosmology (IRGAC) 2006, Barcelona. 


\section{Introduction}

While Einstein's general relativity (GR) has stood all direct experimental tests [1], there are also reasons to try to extend GR. For example the mysterious nature of dark energy and dark matter might become resolved within a modified theory of gravity.

Another reason to try to extend GR is the notion of generality. Within the framework of GR torsion is not included in a natural, geometric way. Indeed any calculation of the connection (either by requiring metric compatibility, or by using the first order formalism) leads to the (symmetric) Levi-Cività connection. One is then free to add torsion, but torsion does not follows naturally from the theory. An interesting generalization of GR would generate torsion in a purely geometric way, analogous to the way the Levi-Cività connection is generated in GR.

The Nonsymmetric Gravitational Theory (NGT) [2] is an extension of GR that drops the standard axiom of GR that the metric is a symmetric tensor. Thus we decompose the general, nonsymmetric metric $g_{\mu \nu}$ in it's symmetric and antisymmetric parts

$$
g_{\mu \nu}=G_{\mu \nu}+B_{\mu \nu}
$$

where $G_{\mu \nu}=g_{(\mu \nu)}, B_{\mu \nu}=g_{[\mu \nu]}$ and $(\cdot)$ and [.] indicate normalized symmetrization and anti-symmetrization, respectively. Indeed there is no physical principle that tells us that the metric should be symmetric and therefore such a generalization is very interesting to study.

Indeed the extra structure of NGT produces interesting results on the issues of dark energy and dark matter [3] [4] [5] and it will also be clear that such a theory produces torsion in a very natural way. Unfortunately the nonsymmetric theory of gravitation suffers from all kinds of problems. The first main problems is the non-uniqueness of the theory, as described in [6]. Since torsion is available and since the linearization procedure is not unambiguous, the final linearized lagrangian is (degenerately) determined by 11 free parameters. The second problem, as described in [7], is the possibility of propagating ghost modes. Fortunately this problem can be relatively easy solved by the introduction of a mass term for the $B$-field [2] [8].

In this talk we consider NGT linearized around a GR configuration. By explicitly constructing two different backgrounds (FLRW-universe and Schwarzschild) we show that the evolution of the $B$-field is unstable. By considering the most general form of the linearized lagrangian, we can explicitly point out which terms cause these instabilities. In [6] it is both shown that these terms cannot be removed and that these terms are not a relic of the linearization. Based on this analysis we are able to write down a consistent, stable linearized lagrangian for the B-field. We next canonically quantize the B-field in inflation and follow its dynamics in radiation and matter era. This analysis shows that the B-field is an excellent dark matter candidate, provided the mass is of the order of the neutrino masses. 


\section{The linearized Lagrangian}

Since GR is very successful, it is natural to assume that any modification of the theory should be relatively small. Therefore we consider NGT in the limit of a small $B$, but an arbitrary $G$. The linearization of the full, general lagrangian is done in Appendix A of [6]. The result is

$$
\begin{aligned}
\mathcal{L}=\sqrt{-G}[ & R+2 \Lambda-\frac{1}{12} H^{2}+\left(\frac{1}{4} m^{2}+\beta R\right) B^{2} \\
& \left.-\alpha R_{\mu \nu} B^{\mu \alpha} B_{\alpha}{ }^{\nu}-\gamma R_{\mu \alpha \nu \beta} B^{\mu \nu} B^{\alpha \beta}\right]+\mathcal{O}\left(B^{3}\right) .
\end{aligned}
$$

Here the curvature terms $R_{\mu \alpha \nu \beta}, R_{\mu \nu}$ and $R$ all refer to the background, GR, curvature. $H_{\mu \nu \rho}$ is the field strength associated with $B_{\mu \nu}$. The coefficients $\alpha, \beta$ and $\gamma$ are determined by the parameters of the 'full' lagrangian and the unambiguous decomposition of the metric in its symmetric and anti-symmetric parts. It is important to note that one cannot consistently choose the parameters of the full theory in such a way that $\gamma=0$ (see appendix A of [6]). The parameters $\alpha$ and $\beta$ can in principle be set to zero, however a priori there is no reason to do this. A mass is naturally generated in the presence of a nonzero cosmological constant and in fact one has

$$
\frac{1}{4} m^{2}=\Lambda\left(\frac{1}{2}-\rho+4 \sigma\right) \propto 10^{-84} \mathrm{GeV}^{2}
$$

where we assume that the parameters $\rho$ and $\sigma$ are order unity. Note that the inequality is not necessarily true at all times, since the cosmological term may change during the evolution of the Universe (for example during phase transitions). The field equations derived from the lagrangian (21) are

$$
\begin{aligned}
& (\sqrt{-G})^{-1} \frac{1}{2} \partial_{\rho}\left(\sqrt{-G} H^{\rho \mu \nu}\right)+\left(\frac{1}{2} m^{2}+2 \beta R\right) B^{\mu \nu} \\
& -\alpha\left(B^{\nu \alpha} R^{\mu}{ }_{\alpha}+B^{\alpha \mu} R_{\alpha}^{\nu}\right)-2 \gamma B^{\alpha \beta} R^{\mu}{ }_{\alpha \beta}{ }^{\nu}+\mathcal{O}\left(B^{2}\right)=0 \\
& R_{\mu \nu}-\frac{1}{2} R G_{\mu \nu}-\Lambda G_{\mu \nu}+\mathcal{O}\left(B^{2}\right)=0 .
\end{aligned}
$$

We see that to this order the field equations decouple and it makes sense to consider the symmetric background, to be just a GR background. The theory then reduces to an antisymmetric tensor field coupled to GR.

\section{Instabilities in NGT}

We first focus on the dynamics of the B-field in an expanding universe§ [9]. Our background metric is given by the (conformal) Friedmann-Lemaitre-Robertson-Walker metric (FLRW):

$$
G_{\mu \nu}=a(\eta)^{2} \eta_{\mu \nu}
$$

where $\eta_{\mu \nu}=\operatorname{diag}(1,-1,-1,-1), \eta$ is conformal time and $a(\eta)$ is the conformal scale factor. The conformal time is related to the standard cosmological time by, $a d \eta=d t$.

$\S$ This section is based on Ref. [6] 
Table 1. The scale factor and conformal time in different eras

\begin{tabular}{|c|c|c|}
\hline era & $a$ & $\eta$ \\
\hline de Sitter inflation & $a=-\frac{1}{H_{I} \eta}$ & $\eta \leq-\frac{1}{H_{I}}$ \\
Radiation & $a=H_{I} \eta$ & $\frac{1}{H_{I}} \leq \eta \leq \eta_{e q}$ \\
Matter & $a=\frac{H_{I}}{4 \eta_{e q}^{2}}\left(\eta+\eta_{e q}\right)^{2}$ & $\eta \geq \eta_{e q}$ \\
\hline
\end{tabular}

The scale factor during the different cosmological eras is given in table 11. where $H_{I} \sim 10^{13} \mathrm{GeV}$ is the Hubble parameter during inflation and $\eta_{e q}$ is the conformal time at matter-radiation equality.

For the following discussion we focus on the 'electric' mode of the B-field: $E_{i} \equiv B_{0 i}$. (the 'magnetic' mode turns out not to be very interesting for our present purpose). If we evaluate the lagrangian (2) and the field equations (41) in the FLRW background we find the follwing equation of motion

$$
\left[\partial_{0} \partial_{0}-\frac{\mathcal{Y}}{\mathcal{X}} \delta^{i j} \partial_{i} \partial_{j}+M_{e f f}^{2}\right] \tilde{E}=0,
$$

where

$$
E=\frac{\sqrt{\mathcal{Y}}}{\mathcal{X}} \tilde{E}
$$

and the effective mass term is given by

$$
M_{\text {eff }}^{2}=-2 \mathcal{Y} a^{2}+\frac{\mathcal{Y}^{\prime \prime}}{2 \mathcal{Y}}-\frac{3\left(\mathcal{Y}^{\prime}\right)^{2}}{4 \mathcal{Y}^{2}} .
$$

Furthermore we have defined

$$
\begin{aligned}
& \mathcal{X}=a^{-2}\left((12 \beta+2 \alpha) \mathcal{H}^{2}+(12 \beta+4 \alpha-2 \gamma) \mathcal{H}^{\prime}-\frac{1}{2} m^{2} a^{2}\right) \\
& \mathcal{Y}=a^{-2}\left((12 \beta+4 \alpha-2 \gamma) \mathcal{H}^{2}+(12 \beta+2 \alpha) \mathcal{H}^{\prime}-\frac{1}{2} m^{2} a^{2}\right)
\end{aligned}
$$

and

$$
\mathcal{H}=\frac{a^{\prime}}{a},
$$

where a prime indicates a derivative with respect to conformal time. We see from (77) that $\tilde{E}$ behaves just as a massive vector field, as long as, $\mathcal{Y} / \mathcal{X}>0$. On the other hand, if $\mathcal{Y} / \mathcal{X}<0$ we see that the spatial derivatives appear with the 'wrong' sign. Since in fourier space these derivatives generate a term proportional to minus the momentum squared, we see that a wrong sign will lead to an exponential growth of the field. Large momenta are no longer suppressed and thus the field will grow without bounds. One could worry about the cases when, $M_{e f f}^{2}<0$. However on dimensional grounds, the effective mass squared scales in the worst case as, $1 / \eta^{2}$. Such a scaling results in a standard power-law enhancement on super-Hubble scales [9] and presents no problem. 


\subsection{Instabilities during Radiation era}

In de Sitter inflation $\mathcal{Y} / \mathcal{X}=1$, and thus the field dynamics are completely regular. However during radiation era we obtain

$$
\left[\partial_{0} \partial_{0}-\frac{H_{I}^{2} m^{2} \eta^{4}+4(\gamma-\alpha)}{H_{I}^{2} m^{2} \eta^{4}-4(\gamma-\alpha)} \delta^{i j} \partial_{i} \partial_{j}+M_{r}^{2}\right] \tilde{E}_{r}=0 .
$$

Here $M_{r}$ is the effective mass during radiation, whose precise form is not important for us. We see however, that we might have problems with the sign of the coefficient in front of the spatial derivatives. For example if we look at the beginning of radiation era $\left(\eta=1 / H_{I}\right)$ we see that if we want $\mathcal{Y} / \mathcal{X}$ to be positive, we need that $m^{2} / H_{I}^{2}$ is at least, $\mathcal{O}(\alpha-\gamma)$. In other words we approximately need:

$$
m \geq|\alpha-\gamma| H_{I} \sim|\alpha-\gamma| \times 10^{13} \mathrm{GeV},
$$

which, unless $|\alpha-\gamma|$ is very small, contradicts Eq. (3). Therefore if we require $\mathcal{Y} / \mathcal{X}$ to be positive, we could drop the purely geometric origin of the lagrangian and add by hand a large $\left(10^{13} \mathrm{GeV}\right)$ mass for the $B$-field, we could fine-tune $\alpha$ or $\gamma$ such that $\alpha-\gamma$ is sufficiently small to satisfy the bound (14), or we could use the more natural requirement that $\alpha=\gamma$. On theoretical grounds only the last of these solutions is satisfactory. A big problem with the first solution is that, while we can always find a

mass where the evolution of the mode is stable, we can than also think of more extreme situations where the mode once again becomes unstable. Therefore we conclude that a natural theory should have $\alpha=\gamma$.

We have also investigated matter era and power-law inflation and we find that similar instabilities are present. However also in these cases $\alpha=\gamma$ stabilizes the system.

\subsection{Instabilities around a Schwarzschild mass}

We have done a similar analysis in a Schwarzschild background. We won't give any details here (see section 4 of [6]), but will only mention that similar instabilities are present; however now the requirements for a stable system are either

$$
\gamma=0
$$

or

$$
m^{2}>\frac{4 \gamma G_{N} \hbar^{2}}{c^{4}} \frac{M_{0}}{r_{0}^{3}} \quad\left[k g^{2}\right]
$$

where we explicitly plugged back factors of $c, h$ and $G_{N} . M_{0}$ is the mass of the object we are considering and $r_{0}$ is the distance where we require stability. for $\gamma$ order 1 this requires e.g. for the exterior of a neutron star $\left(M_{0} \propto M_{\text {sun }}\right.$ and $\left.r_{0} \propto 20 \mathrm{~km}\right)$ :

$$
m \gtrsim \sqrt{|\gamma|} \times 10^{-19} \mathrm{GeV}
$$

However, on theoretical grounds, it is more appealing to require that the $B$-field is stable for all values of $M_{0}$ and $r_{0}$. This can only be achieved if we choose $\gamma=0$. However as noted in section 2, this choice is not possible within our linearization of NGT. 


\section{Antisymmetric metric field as Dark matter}

Based on the previous section we know that the only consistent linearized lagrangian for the $B$-field is

$$
\mathcal{L}=\sqrt{-G}\left[R+2 \Lambda-\frac{1}{12} H^{2}+\left(\frac{1}{4} m^{2}+\beta R\right) B^{2}\right] .
$$

While this lagrangian is not obtainable in NGT, we like to stress that our linearization procedure of NGT lacks any guiding principle (which is reflected in the non-uniqueness of the theory). The analysis above shows that if we want to make sense of nonsymmetric gravity we need to find a guiding principle that, upon linearization, leads to (18). For now we do not know this principle, but we can still study (18). In this section\| we consider the generation and evolution throughout the cosmological history of quantum fluctuations of the $B$-field. In particular we only consider the longitudinal degrees of freedom of the 'magnetic' component [5] 9] [10]

$$
B_{i j} \equiv-\epsilon_{i j k} B_{k}
$$

since this mode gives the dominant contribution to the energy density in the limit $m \rightarrow 0$. For simplicity we take $\beta=0$, but keep the mass arbitrary. When compared to (3) this means we allow the presence of a small bare mass for the $B$-field. In order to quantize the field we perform a Fourier transformation

$$
B^{L}(x)=\int \frac{d^{3} k}{(2 \pi)^{3 / 2}}\left[e^{i \vec{k} \cdot \vec{x}} B^{L}(\eta, \vec{k}) b_{\vec{k}}+e^{-i \vec{k} \cdot \vec{x}} B^{L \star}(\eta, \vec{k}) b_{\vec{k}}^{\dagger}\right],
$$

where $\eta$ is once again conformal time as given in table 1, with canonical commutation relations

$$
\left[b_{\vec{k}}, b_{\vec{k}^{\prime}}^{\dagger}\right]=(2 \pi)^{3} \delta^{3}\left(\vec{k}-\vec{k}^{\prime}\right)
$$

During de Sitter inflation we find that the mode functions approach the conformal vacuum

$$
B_{\mathrm{inf}}^{L} \propto \frac{1}{\sqrt{2 k}} e^{-i k \eta}+\mathcal{O}\left(\frac{m^{2}}{H_{I}^{2}}\right) .
$$

During radiation era the field equations are solved by

$$
B_{\mathrm{rad}}^{L}=\frac{1}{\sqrt{2 k}}\left[\alpha_{\vec{k}}\left(1-\frac{i}{k \eta}\right) e^{-i k \eta}+\beta_{\vec{k}}\left(1+\frac{i}{k \eta}\right) e^{i k \eta}\right]+\mathcal{O}\left(\frac{m^{2}}{H_{I}^{2}}\right)
$$

with the Wronskian condition that

$$
\left|\alpha_{\vec{k}}\right|^{2}-\left|\beta_{\vec{k}}\right|^{2}=1
$$

and we choose $\alpha$ and $\beta$ such that the solutions match at the inflation-radiation transition. Unfortunately we cannot analytically solve the equations of motion in matter era, so there we need to use numerical analysis. We are interested in the power spectrum, which is given by [5]

$$
P_{B}(\vec{k}, \eta)=\frac{H_{I}^{4}}{4 \pi^{2} a^{4}}\left[\left|\partial_{\eta} B_{\vec{k}}^{L}(\eta)+\frac{a^{\prime}}{a} B_{\vec{k}}^{L}(\eta)\right|^{2}+\left(k^{2}+a^{2} m^{2}\right)\left|B_{\vec{k}}^{L}\right|^{2}\right] .
$$

|| Based on Ref. [10] 


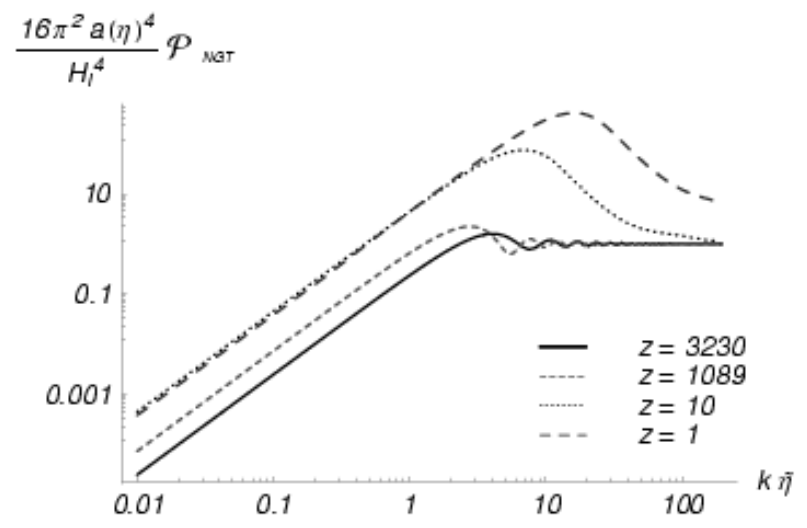

Figure 1. Snapshot of the power spectrum for $m H_{I} \eta_{e q}^{2}=10^{-2}$

A snapshot of this power spectrum, during matter era, for different redshifts is given in figure 1. We find that at late times the power spectrum becomes dominated by a characteristic peak. This peak is caused by modes that are superhorizon $(k \eta \lesssim 1)$ at equality $(z=3230)$, but start to scale as nonrelativistic matter $\left(\propto a^{-3}\right)$ in matter era and enter the horizon. Modes on small enough scales $\left(k \eta>a / a_{e q}\right)$ are effectively massless and scale as relativistic matter $\propto a^{-4}$. The position of the peak is determined by the mass of the $B$-field. In fact we have

$$
k_{\text {peak }}=\sqrt{H_{I} m}
$$

Now that we know the power spectrum, we can calculate the energy density of the $B$-field, defined by

$$
\rho_{B}=\int \frac{d k}{k} P_{B}
$$

A good dark matter candidate should have an energy density

$$
\frac{\rho_{B}}{\rho_{\text {rad }}}=1 \quad \text { at } \quad \eta=\eta_{\text {eq }},
$$

where $\rho_{\text {rad }}$ is the enrgy density of the cosmic radiation. The calculation is done in 10 and it is found that

$$
m=2.8 \times 10^{-2}\left(\frac{10^{13} \mathrm{GeV}}{H_{I}}\right)^{4} \mathrm{eV}
$$

gives the right energy density.

\section{Discussion and conclusion}

We have shown that, while the nonsymmetric theory of gravitation is an extremely interesting extension of general relativity to study, the modes of the antisymmetric metric field are unstable. This instability manifests itself through a wrong sign in front

of spatial derivatives in the equations of motion. Such a wrong sign means that large 
momenta are no longer suppressed, and therefore the field grows without bounds. We showed that the troublesome terms in the lagrangian (2) are the coupling to the Riemann tensor and the Ricci tensor. Furthermore in [6] it was shown that the first of these terms cannot be removed in NGT and that the instabilities are not a relic of the linearization. However, our linearization procedure was rather naive, and it lacks a good guiding principle. Our analysis shows that if one could find a good principle from which to construct a nonsymmetric theory of gravitation (e.g. by considering complex manifolds as in [11] [12]), the linearized lagrangian must have the form of (18). Based on this knowledge, we've studied the evolution of quantum fluctuations, generated at inflation, throughout the cosmological history. We find that the $B$-field has the right energy density to fully take account for the dark matter energy density if the mass of the field is given by $m=2.8 \times 10^{-2}\left(\frac{10^{13} \mathrm{GeV}}{H_{I}}\right)^{4} \mathrm{eV}$. Furthermore the power spectrum develops a characteristic peak, that for this mass and $z=10$ (start of structure formation) has a length scale coincidentally corresponding to the earth sun distance. Although the mass of the $B$-field is small (equivalent to the mass of the $\tau$-neutrino), it still is cold dark matter. Indeed, since the field does not couple to matter fields, it cannot thermalize and therefore the spectrum stays primordial and highly non-thermal. Because of this, it does not suffer from the problems that neutrino dark matter has. As a final remark we note that our dark matter candidate means that gravity may get modified at scales $m^{-1} \propto 0.1 \mu \mathrm{m}\left(\frac{\mathrm{H}_{\mathrm{I}}}{10^{13} \mathrm{GeV}}\right)^{4}$. This is still about two orders of magnitude below the current experimental bound [1].

\section{Acknowledgments}

We would like to thank Wessel Valkenburg and Willem Westra for many interesting discussions and insights on the issue of NGT. Finally we thank John Moffat for his correspondence concerning previous work on NGT

[1] C. M. Will, "The confrontation between general relativity and experiment," gr-qc/0510072

[2] J. W. Moffat, "Nonsymmetric gravitational theory," Phys. Lett. B355 (1995) 447-452, gr-qc/9411006

[3] J. W. Moffat, "Gravitational Theory, Galaxy Rotation Curves and Cosmology without Dark Matter," JCAP 0505 (2005) 003, astro-ph/0412195

[4] J. W. Moffat, "Modified gravitational theory as an alternative to dark energy and dark matter," (2004) astro-ph/0403266

[5] T. Prokopec and W. Valkenburg, "Antisymmetric Metric Field as Dark Matter," astro-ph/0606315

[6] T. Janssen and T. Prokopec, "Instabilities in the nonsymmetric theory of gravitation," Class. Quant. Grav. 23 (2006) 4967-4982, gr-qc/0604094

[7] T. Damour, S. Deser, and J. G. McCarthy, "Nonsymmetric gravity theories: Inconsistencies and a cure," Phys. Rev. D47 (1993) 1541-1556, gr-qc/9207003.

[8] M. A. Clayton, "Massive NGT and spherically symmetric systems," J. Math. Phys. 37 (1996) $395-420, \mathrm{gr}-\mathrm{qc} / 9505005$ 
[9] T. Prokopec and W. Valkenburg, "The cosmology of the nonsymmetric theory of gravitation," Phys. Lett. B636 (2006) 1-4, astro-ph/0503289

[10] W. Valkenburg, "Linearized nonsymmetric metric pertubations in cosmology," master's thesis at the ITP of Utecht University, Available at http://www1.phys.uu.nl/wwwitf/teaching/thesis.htm (2006).

[11] A. H. Chamseddine, "Hermitian geometry and complex space-time," Commun. Math. Phys. 264 (2006) 291-302, hep-th/0503048

[12] R. Mann and J. Moffat, "Ghost properties of generalized theories of gravitation," Phys. Rev. D 26 (1982) 1858. 